\title{
Can current fMRI techniques reveal the micro- architecture of cortex?
}

TO THE EDITOR - In a recent paper in Nature Neuroscience, Kim et al. ${ }^{1}$ claimed to have visualized orientation columns in the visual cortex of cats by means of high-field, spatially resolved functional magnetic resonance imaging (fMRI). This would represent a striking technical advance indeed, if their mapping method were robust to noise and if the recorded patterns were reproducible within a given experimental session. A careful examination of their data, however, suggests that this may not be the case. Because the maps obtained in this study rely on a specific phase of the hemodynamic response, I will begin by briefly reviewing a few basic principles.

When a stimulus of a few seconds' duration is presented while $\mathrm{T} 2{ }^{*}$-weighted images are being continuously recorded, the evolution of activation has at least two phases: an increase in intensity, usually referred to as overcompensation, and an undershoot with a long time constant following the offset of the stimulus ${ }^{2}$. In addition, some fMRI time series show an initial, transient signal loss (dip) starting almost immediately after stimulus presentation and lasting for about four to five seconds ${ }^{3-5}$. This peculiar bi- or triphasic evolution of the stimulus-induced activation occurs because the fMRI signal is sensitive to hemodynamic changes rather than to changes in neural activity per se. The commonly used blood oxygen level-dependent (BOLD) contrast signal, for instance, reflects changes in blood volume, flow and oxygen concentration. The early dip is thought to reflect a localized increase of deoxyhemoglobin in the parenchyma, whereas the later positive phase is believed to stem from an increase in cerebral blood volume and flow that extends considerably beyond the site of increased neural activity and tends to overcompensate for the increase in oxygen consumption ${ }^{6}$.

Kim et al. ${ }^{1}$ generated maps of orientation columns in area 18 of the cat by exploiting this localized dip. Specifically, they presented cats with oriented, drifting gratings, and determined the stimulusinduced activation by monitoring any negative signal the absolute value of which was greater than $(0.5+1 \mathrm{~s} . \mathrm{ds}) \%$ at 2 seconds after stimulus onset (Fig. 1d of ref.1). They also obtained a conventional activation map (Fig. 1c in ref. 1) by correlating the time course of the hemodynamic response with that of the visual stimulus under exactly the same stimulus conditions in the same session.

Assuming that the dip is due to a local increase of deoxyhemoglobin, one would obviously expect every single time series exhibiting this early negativity to exhibit the subsequent overcompensation as well. In fact, optical imaging experiments have never detected an initial negativity that was not followed by the typical overcompensation (A. Grinvald, personal communication). Furthermore, if the dip reflects—as it should-a signal that is spatially restricted within the parenchyma, one would also expect it to occur almost exclusively outside the areas transpierced by major drainage vessels, such as the sagittal sinuses; this is particularly true of the very early (two-second) phase of the negativity that was analyzed by Kim et al. ${ }^{1}$ Yet an exact superposition of Figs. 1c and 1d of ref. 1 shows that the above assumptions do not hold for their data (Fig. 1 below). The areas showing a dip largely overlap with the sinuses, and a dip signal can be also detected in areas showing no statistically significant conventional activation. The former is puzzling because studies in humans by other investigators from the same laboratory ${ }^{5}$, as well as studies in monkeys ${ }^{4}$, have shown that large vessels like the sinus show only positive signal changes. The latter is also puzzling, because the dip is far smaller than the conventional activation signal and should therefore be more difficult to detect in noisy images.

A logical conclusion from these observations is that the initial dip, especially when analyzed with the simple statistics used by Kim et al. ${ }^{1}$, yields maps that are extremely sensitive to noise and that may have very little to do with the actual columnar architecture of the cortex. An obvious test for reliability would be to repeat the acquisition of maps for a given orientation within a single session and to ensure that they are reproducible. Unfortunately, the authors have not reported any such tests. It should be noted, however, that repeated acquisitions in our own laboratory (unpublished-and ultimately unpublishable-data) using almost the same hardware, the same spatial resolution and very similar postprocessing techniques failed to reveal an acceptable consistency in dip-derived ocular dominance maps of the monkey visual cortex. Patterns that resembled columnar organization were found occasionally, but they were not reproducible within a single session and were therefore discarded.

\section{Nikos Logothetis}

Max-Planck Institute for Biological Cybernetics, Spemannstr. 38, 72076 Tübingen, Germany email: nikos.logothetis@tuebingen.mpg.de

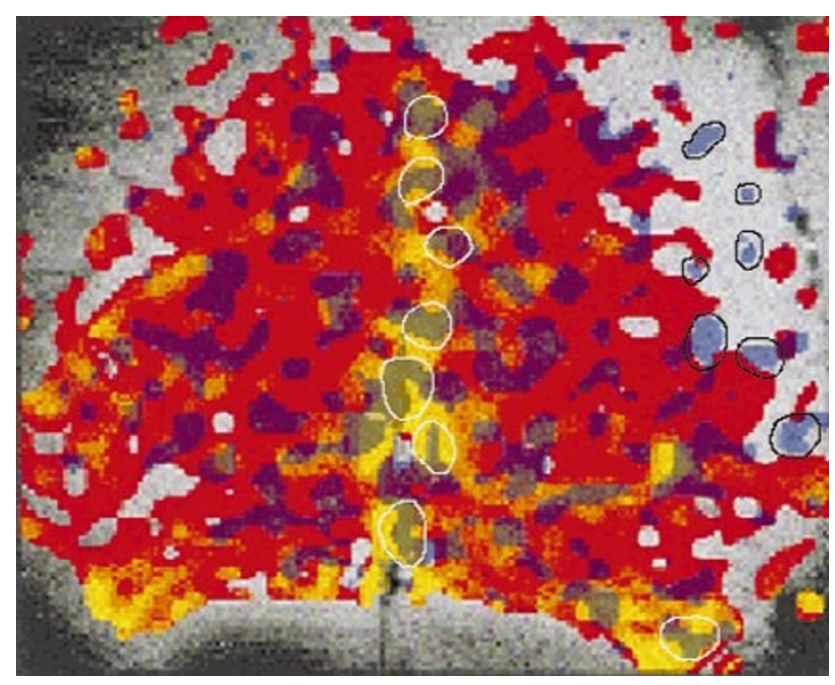

Fig. I. The activity derived by detecting the initial dip superimposed on the activity mapped by correlating the time series of each voxel with the stimulus time course (data from ref. I). White contours indicate some dip-determined areas that completely overlap with the sagittal or horizontal sinuses. Black contours indicate some other dip-determined activity in regions showing no significant positive activation. 
REPLY - In our recent article ${ }^{1}$, we reported the use of high spatiotemporal resolution BOLD fMRI to detect early-negative BOLD signal changes and its application to map submillimeter iso-orientation columns in the cat visual cortex. Because the early-negative BOLD contrast is small and transient, the authenticity of the columnar layout derived from these data has been questioned by Dr. Logothetis. We would like to respond to his criticisms point by point.

Do functional maps based on the early-negative BOLD responses contain pixels from noise fluctuations? The answer is of course yes. In most functional data analysis methods, an arbitrary statistical threshold (that is, $p$ value) is used to generate a functional map. Based on the statistical threshold used in our analysis, $\sim 90 \%$ of the active pixels in the singlecondition map (Fig. 1d in ref. 1) are expected to be caused by the visual stimulation, whereas the remaining $\sim 10 \%$ of active pixels arise from random noise fluctuations. For example, the small number of early-negative response pixels without subsequent positive overcompensation (black contours in Fig. 1 above) can be attributed to noise fluctuation. The use of more sophisticated statistical methods might help to minimize noise contibution.

Why are the early-negative BOLD pixels also found around the sagittal sinus area? Functional MRI does not detect neural activity per se, but rather the resulting change in blood deoxyhemoglobin concentration. Deoxyhemoglobin drains from capillaries into venules and eventually into large veins. In $\mathrm{fMRI}^{7}$ and optical imaging ${ }^{6}$ studies, the negative BOLD signal changes induced by increase of deoxyhemoglobin concentration were observed initially in the active parenchyma, and later in the draining veins. Therefore, the presence of some negative response pixels around the sinus is not surprising. However, in our study, the negative response pixels around the sinus are clustered in patchy domains (Fig. $1 \mathrm{~d}$ of ref. 1 and white contours in Fig. 1 above), in contrast to Logothetis' statement that these negative response pixels are 'largely overlapping' with the boundaries of the sagittal sinus. This, plus the fact that our imaging slice was $\sim 0.5 \mathrm{~mm}$ below the cortical surface, indicates that the negative response pix- els around the sinus originated predominantly from tissue areas (for example, columns from the suprasplenial gyrus on the medial bank) below the sagittal sinus. Nevertheless, our results clearly contradict the previous fMRI studies in monkeys by Logothetis et al. ${ }^{4}$, which claimed that the early-negative BOLD signal change was observed only in the tissue, and not in the sagittal sinus (Fig. 5 in ref. 4). This is puzzling, because optical imaging studies suggest that deoxyhemoglobin signals should be observed also in large draining vessels ${ }^{3}$. More importantly, in their paper $^{4}$, the onset time $(6 \mathrm{~s})$ of positive BOLD signals from the tissue significantly lags behind the onset time (1 s) of positive signals from the sagittal sinus (Fig. $5 \mathrm{c}$ in ref. 4). This is counter-intuitive; given that blood drains from tissue to large veins, the signal in the tissue ought to precede the signal in the sinus. One possible explanation for the discrepency between our data ${ }^{1}$ and Logothetis et al. ${ }^{4}$ is that the signals that Logothetis et al. ${ }^{4}$ attribute to neural activation in their study might have been contaminated by large-vessel flow effects due to rapid radiofrequency pulsing; this so-called 'inflow effect' is a wellknown source of artifact in the fMRI community ${ }^{8}$.

Are the columnar maps obtained from the negative BOLD signals genuine? Although our early-negative BOLD maps contain some pixels both from noise and from draining-vessel artifacts, the overall functional specificity of columnar maps is convincing. Unlike the positive BOLD response, the early-negative response is highly specific to the cortical columns (Fig. 2 in ref. 1). For example, the $45^{\circ}$ columns have negative BOLD responses during the $45^{\circ}$ stimulus, but not during the $135^{\circ}$ (orthogonal) stimulus. More importantly, as noted in the accompanying News and Views by Grinvald et al. ${ }^{6}$, the single-condition columnar maps of orthogonal orientations $\left(0^{\circ}\right.$ versus $90^{\circ}$ and $45^{\circ}$ versus $135^{\circ}$ ) are complementary. Moreover, topological features of the composite maps obtained from the negative BOLD signals (Fig. 4a in ref. 1) are consistent with those seen with optical imaging and single-neuron recording. Taken together, our results strongly support the authenticity of columnar architectures derived from the negative BOLD signals.
To further corroborate the functional validity of negative BOLD signals, more experiments are necessary, as discussed by Grinvald et al. ${ }^{6}$ and by Logothetis in his letter. We are actively working to improve the contrast-to-noise ratio, the reproducibility of single-condition columnar maps, and the cross-validation of fMRI maps with single and multi-neuron recordings.

Much work needs to be done before the early-negative BOLD technique can be applied for routine brain mapping at columnar resolution, especially in humans. The most important challenges are to increase the detectability of the negative BOLD signals (for example, by using high magnetic fields and better data processing methods) and to eliminate large vessel contributions (for example, by using different fMRI approaches such as spin-echo ${ }^{9}$ and perfusion-based ${ }^{10} \mathrm{MRI}$ techniques). As with any new technique, this novel method for non-invasive visualization of cortical columns by fMRI will need further improvement and cross-validation. We believe, however, that our results lay the foundation for non-invasive mapping of brain functions at columnar resolution, thus opening new vistas for neuroscience research.

\section{Dae-Shik Kim, Timothy Q. Duong and Seong-Gi Kim}

Center for Magnetic Resonance Research, University of Minnesota Medical School, Minneapolis, Minnesota 55455, USA

email (S.-G.K.): kim@cmrr.umn.edu

1. Kim, D-S., Duong, T. Q. \& Kim, S.-G. Nat. Neurosci. 3, 164-169 (2000).

2. Kruger, G., Kleinschmidt, A. \& Frahm, J. Magn. Reson. Med. 35, 797-800 (1996).

3. Hu, X., Le, T. H. \& Ugurbil, K. Magn. Reson. Med. 37, 877-884 (1997).

4. Logothetis, N. K., Guggenberger, H., Peled, S. \& Pauls, J. Nat. Neurosci. 2, 555-562 (1999).

5. Menon, R. S. et al. Magn. Reson. Med. 33, 453-459 (1995).

6. Grinvald, A., Slovin, H. \& Vanzetta, I. Nat. Neurosci. 3, 105-107 (2000).

7. Duong, T.Q., Kim, D.-S., Ugurbil, K. \& Kim, S.-G. Magn. Reson. Med. in press (2000).

8. Kim, S.-G. \& Ugurbil, K. J. Neurosci. Methods 74, 229-243 (1997).

9. Lee, S.-P., Silva, A.C., Ugurbil, K. \& Kim, S.-G. Magn. Reson. Med. 42, 919-928 (1999).

10. Kim, S.-G. Magn. Reson. Med. 34, 293-301 (1995). 